ARTICLE OPEN

\title{
Prediction of crossing nodal-lines and large intrinsic spin Hall conductivity in topological Dirac semimetal $\mathrm{Ta}_{3} \mathrm{As}$ family
}

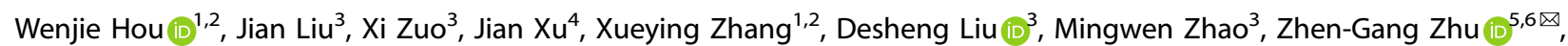 \\ Hong-Gang Luo $\mathbb{B}^{4,7 凶}$ and Weisheng Zhao $\mathbb{D}^{1,2 \bowtie}$
}

Topological insulators (Tls) are considered as ideal platforms for generating large spin Hall conductivity (SHC), however, the bulk carrier problem, which is unavoidable in Tls, hinders their practical applications. Recently, topological semimetals (TSMs) have been proposed to achieve large SHC to replace Tls. However, the ideal TSM candidates with large SHC are still lacking. In terms of firstprinciples calculations, we predict that $\mathrm{Ta}_{3}$ As family compounds exhibit complex crossing nodal-lines (CNL) properties in absence of the spin-orbit coupling (SOC). However, they transfer to Dirac TSMs under the influence of strong SOC, and present large SHC around Fermi level in particular. Remarkably, the $\mathrm{SHC}$ value of $\mathrm{Ta}_{3} \mathrm{Y}(\mathrm{Y}=\mathrm{As}, \mathrm{Sb}, \mathrm{Bi})$ is around $1500-1700(\hbar / e)(\Omega \cdot \mathrm{cm})^{-1}$, which is comparable to noble metal Pt and much larger than TIs, Weyl TSMs, and $4 d / 5 d$ transition metals. Our work not only suggests a kind of TSM family with interesting Dirac CNL around Fermi level, but also paves the way for searching large intrinsic SHC materials in complex CNL TSM systems.

npj Computational Materials (2021)7:37; https://doi.org/10.1038/s41524-021-00504-w

\section{INTRODUCTION}

The spin Hall effect (SHE) is a phenomenon that a transverse pure spin current appears if a longitudinal electric field is applied ${ }^{1-4}$. Topological insulators (TIs) and quantum SHE systems were considered as ideal materials for producing pure spin current ${ }^{5,6}$. However, the experiments have shown that the bulk carrier problem prevents the widespread applications of Tls in spintronic devices because of the hybridization between surface states and bulk states in $\mathrm{Bi}_{2} \mathrm{Se}_{3}$ class materials ${ }^{7,8}$. Generally speaking, there are two kinds of mechanisms for the SHE, which are the extrinsic mechanism and intrinsic mechanism. For the former, impurities and defects with strong SOC are the main scattering resources. Both the side-jump and skew scattering are specifically identified and similar to those in the study of anomalous Hall effect. The intrinsic mechanism roots in the topologically nontrivial energy bands, which can be accurately calculated based on ab initio calculations and Kubo formula. Recently, heavy metals and compounds including heavy elements have attracted growing attention for the possibility to realize large $\mathrm{SHE}^{9-13}$. In particular, a lot of interests focus on topological semimetals (TSMs), which exhibit similar spin-momentum locking in both bulk and topological surface states. To date, the SHC of some TSMs, like TaAs, NbAs, and rutile oxides ${ }^{9,14}$, is still far below that of pure heavy metal Pt and W. Therefore, the TSMs with larger SHC are still highly desirable both for the purposes of fundamental research and technical applications.

According to the distribution and degeneracy of crossing nodal points, TSMs can usually be divided into three categories: Dirac semimetals, Weyl semimetals, and nodal line semimetals $(\mathrm{NLSMs})^{15-23}$. The Weyl points are discrete points in momentum space where the conduction bands and valence bands cross each other. However, in the NLSMs, these crossing points are not discrete but form a continuous path. Compared to the Fermi arc surface states existing in Dirac and Weyl semimetals, NLSMs show drumhead surface states, which are regarded as excellent platforms hosting many interaction-induced nontrivial states, such as superconductivity and fractional topological insulator ${ }^{21}$. It is known that the nodal line can be protected by appropriate symmetries, such as mirror symmetry, time-reversal symmetry, and inversion symmetry ${ }^{24-26}$. Interestingly, the nodal line states have been classified into various types, e.g., isolated closed nodal ring, nodal chain, nodal link, nodal knots, nodal straight line, and even crossing nodal-line $(\mathrm{CNL})^{27,28}$. Until now, the real NLSMs that can host CNL are very rare ${ }^{28}$. It is curious to find these CNL semimetals (CNLSMs) and explore their possibility on exotic SHC applications.

In this article, using first-principles calculations, we propose that the $X_{3} Y(X=T a, N b ; Y=A s, S b, B i)$ compounds are a family of CNLSMs. Interestingly, without taking into account the effects of SOC, $\mathrm{X}_{3} \mathrm{Y}$ compounds host two sets of complex CNL around Fermi level. One is located around the corners, while others are distributed in the bulk of momentum space. When the SOC is present, Dirac points are emergent along the R-M path due to the $C_{4}$ rotational symmetry and are absent along other directions with a very tiny gap. Importantly, it is found that $\mathrm{Ta}_{3} \mathrm{Y}$ compounds exhibit large intrinsic SHC. For example, the SHC is around 1500 $(\hbar / e)(\Omega \cdot \mathrm{cm})^{-1}$ in stable $\mathrm{Ta}_{3}$ As compound, which is attributed to its large SOC band splitting. Therefore, the prediction of Dirac CNLSMs in $\mathrm{X}_{3} \mathrm{Y}$ family not only extends our current knowledge on NLSMs, but also suggests a routine of realizing large SHC to replace noble metals. Significantly, $X_{3} Y$ family materials, in particular the stable $\mathrm{Ta}_{3} \mathrm{As}$ compound, are promising candidates for the application in spintronic devices ${ }^{29-32}$.

\footnotetext{
${ }^{1}$ Fert Beijing Institute, MIIT Key Laboratory of Spintronics, School of Integrated Circuit Science and Engineering, Beihang University, Beijing, China. ${ }^{2}$ Beihang-Goertek Joint Microelectronics Institute, Qingdao Research Institute, Beihang University, Qingdao, China. ${ }^{3}$ School of Physics, State Key Laboratory of Crystal Materials, Shandong University, Jinan, China. ${ }^{4}$ Beijing Computational Science Research Center, Beijing, China. ${ }^{5}$ School of Electronic, Electrical and Communication Engineering, University of Chinese Academy of Sciences, Beijing, China. ${ }^{6}$ CAS Center for Excellence in Topological Quantum Computation, University of Chinese Academy of Sciences, Beijing, China. ${ }^{7}$ School of Physical Science and Technology, Lanzhou University, Lanzhou, China. ${ }^{凶}$ email: zgzhu@ucas.ac.cn; luohg@lzu.edu.cn; weisheng.zhao@buaa.edu.cn
} 


\section{RESULTS AND DISCUSSION}

\section{Crystal structures and stability of $X_{3} Y$ compounds}

The crystal structure of the $X_{3} Y(X=T a, N b ; Y=A s, S b, B i)$ compounds is the same as the ordered $L 12$ phase $\mathrm{Cu}_{3} \mathrm{Au}^{16,33}$. We adopt the cubic structure with the space group $\operatorname{Pm} \overline{3} m$ (No. 221), as shown in Fig. 1a. A primitive cell contains three $X$ atoms at the plane center and one $Y$ atom at the vertex. Figure $1 \mathrm{~b}$ shows the bulk Brillouin zone (BZ) of the $\mathrm{X}_{3} \mathrm{Y}$ and the projected surface $\mathrm{BZ}$ of the (010) surface. Previous studies mainly focused on the $\mathrm{Ta}_{3} Y$ compounds with the $\mathrm{Ti}_{3} \mathrm{P}$-type (A15 phase, space group 223) structure ${ }^{34,35}$, instead of the $\mathrm{Pm} \overline{3} m$ phase. To illustrate the thermodynamic and lattice stability of $P m \overline{3} m$-phase $X_{3} Y$ compounds, we calculate their formation enthalpies $(\Delta H)$ and the phonon spectra. The negative $\Delta H$ values of $\mathrm{Ta}_{3} \mathrm{As}, \mathrm{Nb}_{3} \mathrm{As}$, and $\mathrm{Nb}_{3} \mathrm{Sb}$ indicate that they are thermodynamically stable against phase decomposition, as shown in Fig. 1c. In addition, the absence of imaginary modes in the entire BZ further confirms their lattice stability, as shown in Supplementary Fig. 1. The calculated lattice constant of $\mathrm{Ta}_{3} \mathrm{As}, \mathrm{Nb}_{3} \mathrm{As}$, and $\mathrm{Nb}_{3} \mathrm{Sb}$ are $4.102,4.105$, and $4.226 \AA$, respectively.

\section{Electronic structures and effective Hamiltonian model}

The calculated electronic structures of $\mathrm{Ta}_{3} \mathrm{As}, \mathrm{Nb}_{3} \mathrm{As}$, and $\mathrm{Nb}_{3} \mathrm{Sb}$ present similar characters, with fourfold degeneracy around a crossing point because of the time-reversal and inversion symmetry. Without the SOC effects, Dirac points exist along high-symmetry paths in the $B Z$, e.g., $M-X$ and $M-\Gamma$ paths, as shown in Fig. 2. Similar to the case of $\mathrm{Cu}_{3} \mathrm{PdN}$ and Mackay-Terrones crystal, the band crossings can be understood from the argument of codimension ${ }^{16-21}$. In general, the four bands without SOC effects near the Dirac points can be described by two identical $2 \times 2$ Hamiltonians, which are written as

$H(k)=d_{x}(k) \sigma_{x}+d_{y}(k) \sigma_{y}+d_{z}(k) \sigma_{z}$

Where $\sigma_{x, y, z}$ and $d_{x, y, z}(k)$ are Pauli matrices and corresponding coefficients, respectively. At $\Gamma$ and $R$ point with little group $O_{h}$, the time-reversal, inversion symmetry as well as rotation symmetry restrict the coefficients, with $d_{y}(k)=0, d_{x}(k)$, and $d_{z}(k)$ being odd and even functions correspondingly. We can write $d_{x, z}(k)$ as follow,

$$
\begin{aligned}
& d_{x}(k)=\gamma k_{x} k_{y} k_{z}+\lambda_{z} k_{x}^{2} k_{y}^{2} k_{z}+\lambda_{y} k_{x}^{2} k_{y} k_{z}^{2}+\lambda_{x} k_{x} k_{y}^{2} k_{z}^{2}+\ldots \\
& d_{z}(k)=M-B\left(k_{x}^{2}+k_{y}^{2}+k_{z}^{2}\right)
\end{aligned}
$$

Here $\gamma, \lambda_{x, y, z}, M$, and $B$ are the corresponding coefficients. The eigenvalue of Eq. (1) is $E(k)= \pm \sqrt{d_{x}^{2}(k)+d_{z}^{2}(k)}$. The band $\mathbf{a}$

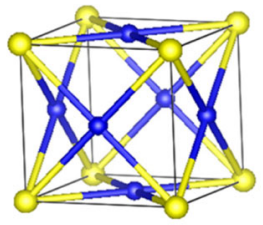

- $\mathrm{Ta}, \mathrm{Nb} \bigcirc \mathrm{As}, \mathrm{Sb}, \mathrm{Bi}$

b

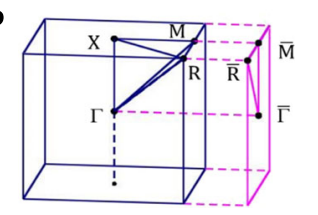

c

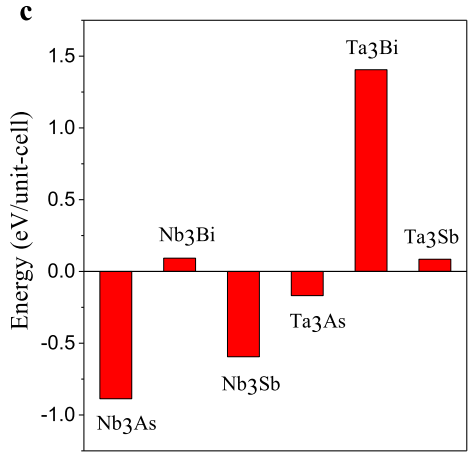

Fig. 1 Crystal structure. a The atomic structure of the L12 phase $\mathrm{X}_{3} \mathrm{Y}(\mathrm{X}=\mathrm{Ta}, \mathrm{Nb} ; \mathrm{Y}=\mathrm{As}, \mathrm{Sb}, \mathrm{Bi})$ family compounds. Blue and yellow spheres denote the $X$ and $Y$ atoms, respectively. $\mathbf{b}$ The bulk Brillouin zone (BZ) and the projected BZ on the (010) surface. $c$ The formation enthalpies $(\Delta H)$ of different $\mathrm{X}_{3} \mathrm{Y}$ compounds. The unit is eV per unitcell. crossing points appear only for the case of $E(k)=0$, i.e., both $d_{x}(k)=0$ and $d_{z}(k)=0$. Here, the condition $d_{z}(k)=0$ is satisfied only when energy bands inversion occurs. As shown in Supplementary Fig. 2, it is clear that the $p$ - $d$ bands inversion happens along the $\mathrm{R}-\Gamma, \mathrm{R}-\mathrm{X}$, and $\mathrm{R}-\mathrm{M}$ paths. Furthermore, if we ignore the higher-order terms in Eq. (2) with $d_{x}(k)=\gamma k_{x} k_{y} k_{z}=0$, Eq. (2) and Eq. (3) induce a nodal line topological state ${ }^{16}$.

However, the crossing points are gapped along $M-X, M-\Gamma, R-\Gamma$, and $\mathrm{R}-\mathrm{X}$ paths but preserved along $\mathrm{R}-\mathrm{M}$ path when taking into account the SOC in Fig. 2. The band structures are similar for $\mathrm{Ta}_{3} \mathrm{As}, \mathrm{Nb}_{3} \mathrm{As}$, and $\mathrm{Nb}_{3} \mathrm{Sb}$. Clearly, both the $\mathrm{R}$ and $\Gamma$ points belong to the $O_{h}$ double point group, and the irreducible representation of bands near the Dirac points is marked with the red symbol $\Gamma_{i}$ $(i=4,5,6,7)$. (i) The R-X path: Moving from $\mathrm{R}$ to $\mathrm{X}$, the symmetry is lowered to $C_{2 v}$ double point group, where only the $\Gamma_{5}$ irreducible representation exists ${ }^{36}$. (ii) The R- $\Gamma$ path: Characterized by $C_{3 v}$ double group, both the occupied band and non-occupied one along $\mathrm{R}-\Gamma$ path are the $\Gamma_{4}$ irreducible representation in $C_{3 v}$ double point group. As we know, if the two bands at the crossing points belong to the same irreducible representation, the crossing points will not be protected by the symmetry ${ }^{37}$. In Fig. 2a (without SOC) and Fig. $2 \mathrm{~d}$ (with SOC), it is observed that the crossing points are gapped with a tiny value along $\mathrm{R}-\mathrm{X}$ and $\mathrm{R}-\Gamma$ path due to the effect of SOC. (iii) The R-M path: The crossing points still exist, which results from the non-occupied and occupied bands belonging to different irreducible representations $\Gamma_{6}$ and $\Gamma_{7}$ in the $C_{4 v}$ double point group, respectively. The existing crossing points are protected by crystal symmetry, thus no gap appears along the $\mathrm{R}-\mathrm{M}$ path. To express the influence of SOC strength on Dirac points, we take $\mathrm{Ta}_{3} \mathrm{As}$ as an example to show the evolution of energy bands at different SOC strengths along the R-X and R-M path in Supplementary Fig. 4. When the SOC strength is artificially increased to three times larger than the intrinsic value, the Dirac points disappear obviously along the $\mathrm{R}-\Gamma$ path but still exist along the R-M path.

\section{Crossing nodal-lines (CNL) and drumhead surface states}

In previous research for Dirac nodal line and nodal sphere, the energy gap is $E_{g}<2 \mathrm{meV}^{28}$. This energy scale is rough and it is desirable to explore the behavior of Dirac nodal points more precisely. Therefore, we explore extremely tiny energy gaps which are identified at $0.1 \mathrm{meV}$ level, and present their distributions in 3D momentum space as well as the topological surface states in Fig. 3. It is clearly seen that continuous Dirac nodal points are merging into nodal lines, and they cross each other forming the crossing nodal-lines. Moreover, three nodal lines intersect at the $R$ point, while two lines form a nodal net centered on the $\Gamma$ point. And because of the same $O_{h}$ double group at both points, the crossing nodal-lines appear at the position of the $R$ point and $\Gamma$ point. As we know, the NLSMs should present drumhead surface states, which can be observed in Fig. $3 \mathrm{~b}$ with the connection between conduction and valence bands on the (010) surface ${ }^{21}$. Besides, the surface states on other oriented surfaces, like (100) and (001) surface, are also calculated, as shown in Supplementary Fig. 7. It is observed that the drumhead surface states on the above three surfaces are almost the same, which is similar to the case in $\mathrm{YH}_{3}$ materials ${ }^{28}$.

\section{Spin Hall conductivity}

As a measurable and calculated physical quantity, the intrinsic spin Hall conductivity $(\mathrm{SHC})$ is usually used to describe the strength of the SHE. The SHC and SHE in TSMs have been explored in a large number of previous works. It is indicated that band crossings may lead to large $\mathrm{SHC}^{9,14}$. In view of band characters, we also find and analyze large SHC around the Fermi energy in the $\mathrm{Ta}_{3} \mathrm{Y}(\mathrm{Y}=\mathrm{As}$, Sb, Bi) compounds. At the zero-temperature limit, the 

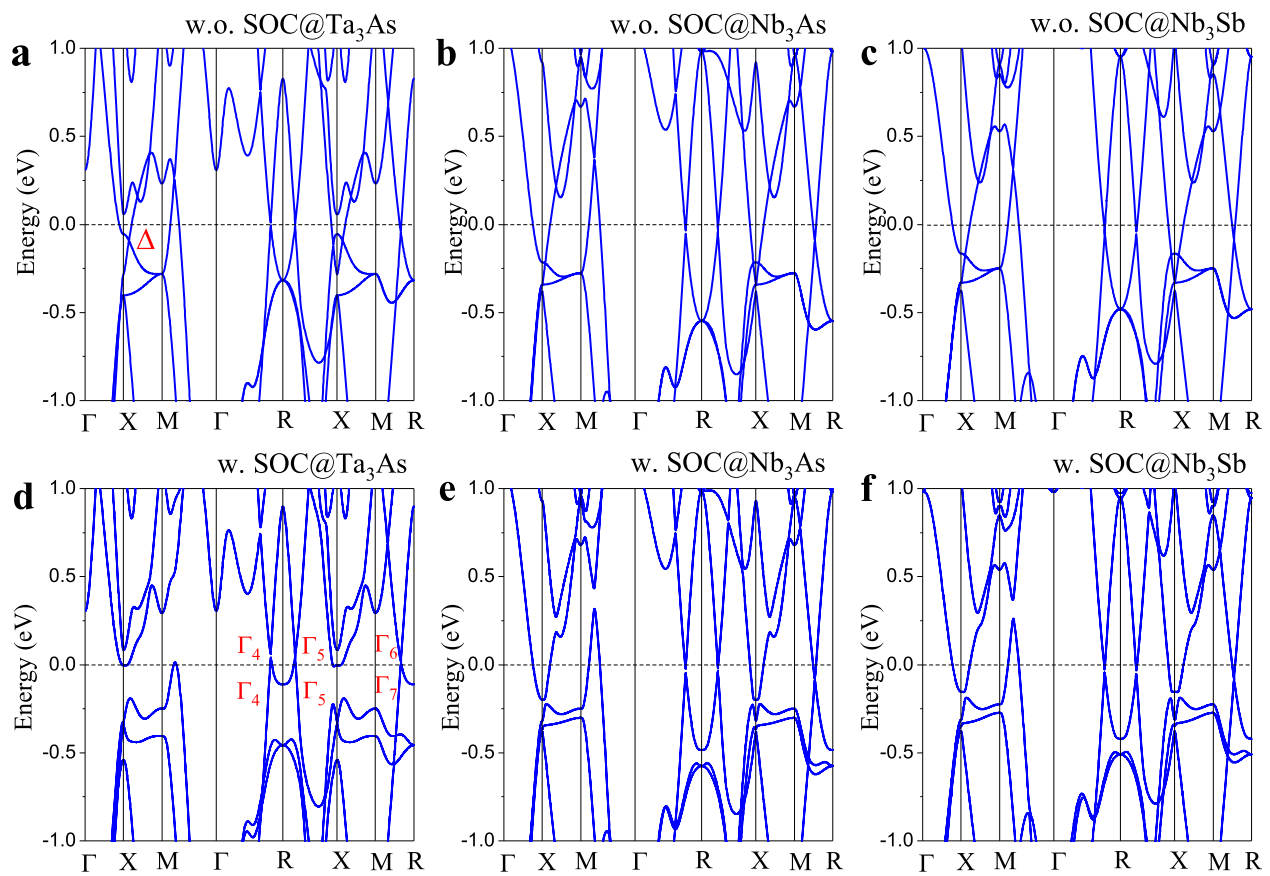

Fig. 2 Calculated band structure of $\mathrm{X}_{3} \mathrm{Y}$ family materials without and with spin-orbit coupling (SOC). $a$, $\mathbf{d}$ Band structure of Ta ${ }_{3} A s$ without and with $\mathrm{SOC}$ (the left column); $\mathbf{b}$, e That of $\mathrm{Nb}_{3} \mathrm{As}$ (the middle column); $\mathbf{c}$ and $\mathbf{f}$ That of $\mathrm{Nb}_{3} \mathrm{Sb}$ (the right column). Here, the position of point $\Delta$ (panel a) related to band inversion contributes to the large SHC.
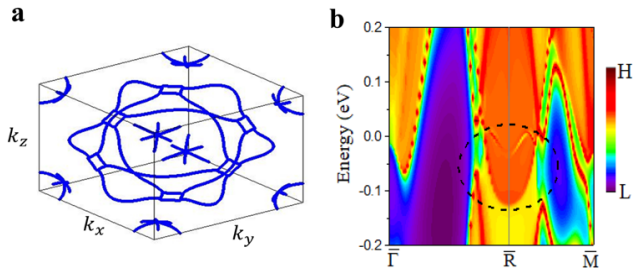

Fig. 3 The crossing nodal-lines and topological surface states. a The distribution of the Dirac points without spin-orbit coupling in the first Brillouin zone. Crossing nodal-lines are observed. $\mathbf{b}$ The drumhead surface state on the (010) surface at the R point around the Fermi level. The spin-orbit coupling is included.

intrinsic SHC tensor can be expressed using the Kubo formula ${ }^{38,39}$,

$\sigma_{x y}=\frac{\hbar}{V N_{k}^{3}} \sum_{k} \sum_{n} f_{n k} \times \sum_{m \neq n} \frac{2 \operatorname{lm}\left[\left\langle n k\left|\hat{J}_{x}\right| m k\right\rangle\left\langle m k\left|-e \hat{v}_{y}\right| n k\right\rangle\right]}{\left(\epsilon_{n k}-\epsilon_{m k}\right)^{2}-(\hbar \omega+i \eta)^{2}}$

Here, $\hat{j}_{x}=\frac{1}{2}\left[\hat{s}_{Z}, \hat{v}_{x}\right]$ is the spin current operator, $V$ is the primitive cell volume, $N_{k}^{3}$ is the number of $k$ points in the BZ, $n(m), \epsilon_{n}$ and | $m k\rangle$ are band indexes, eigenvalues and the Bloch wave function, respectively, $f_{n k}$ is the Fermi distribution function, and $\hat{v}_{y}=\frac{1}{\hbar} \frac{\partial H(k)}{\partial k_{y}}$ is the velocity operator. Besides, we multiply a factor $2 e / \hbar$ to the SHC in the unit of charge conductivity $(\Omega \cdot \mathrm{cm})^{-1}$, similar to anomalous Hall conductivity ${ }^{40}$.

The SHC with the chemical position for the $X_{3} Y$ family is displayed in Fig. 4a. Obviously, the SHC reaches its peak value of about $1300-1700(\hbar / e)(\Omega \cdot \mathrm{cm})^{-1}$ just near the Fermi energy. In comparison, the $\mathrm{SHC}$ of $\mathrm{Ta}_{3} \mathrm{As}$ is approximate $1500(\hbar / e)(\Omega \cdot \mathrm{cm})^{-1}$, which is much larger than that of GaAs compounds about $100(\hbar / e)(\Omega \cdot \mathrm{cm})^{-1}, W_{T}<400$ $(\hbar / e)(\Omega \cdot \mathrm{cm})^{-1}, \mathrm{OsO}_{2} \sim 541(\hbar / e)(\Omega \cdot \mathrm{cm})^{-1}$, and $\mathrm{TaAs} \sim 781$ $(\hbar / e)(\Omega \cdot \mathrm{cm})^{-1}$, as well as $4 d$ and $5 d$ transition metals $<1000$ $(\hbar / e)(\Omega \cdot \mathrm{cm})^{-1}$ and $\beta-\mathrm{W} \sim 1255(\hbar / e)(\Omega \cdot \mathrm{cm})^{-1[9-12,14,39,41}$, even comparable to that of Pt $\sim 2200(\hbar / e)(\Omega \cdot \mathrm{cm})^{-1[10}$. Thus, $\mathrm{Ta}_{3} \mathrm{Y}$ compounds belong to a kind of Dirac CNLSMs with large
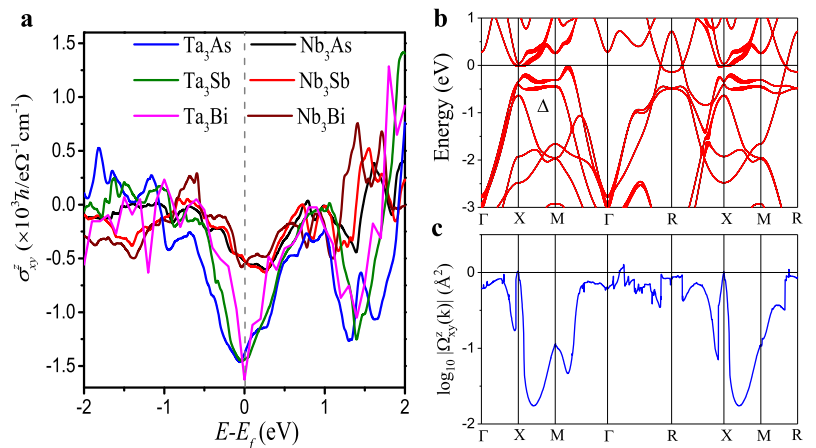

Fig. 4 The intrinsic spin Hall conductivity (SHC). a The SHC $\sigma_{x y}^{z}$ as a function of the chemical position. The gray vertical line corresponding to the Fermi energy. Lines in different colors represent the SHC of different CNLSMs $\mathrm{X}_{3} \mathrm{Y}$ compounds. $\mathbf{b}$ The band-resolved SHC of $\mathrm{Ta}_{3}$ As. c The momentum-resolved $\mathrm{SHC}$ of $\mathrm{Ta}_{3} \mathrm{As}$ along the highsymmetry momentum paths.

SHC, which could be applied to spintronic devices such as the magnetic tunnel junction based on spin-orbit torque ${ }^{29}$.

Further, we analyze the mechanism by calculating the bandresolved and momentum-resolved $\mathrm{SHC}$ of the stable $\mathrm{Ta}_{3} \mathrm{As}$ compound, corresponding to Fig. $4 \mathrm{~b}$, c. Here, the contribution to SHC is noted with red color dots in Fig. $4 \mathrm{~b}$, and the momentumresolved SHC after taking the logarithm is shown in Fig. 4c. From Fig. 4 and Fig. 2 as well as Supplementary Fig. 5, we observe that the states around $\Delta$ point (along $\mathrm{X}-\mathrm{M}$ path) mostly contribute to the large SHC with strong SOC effect. Note that the SHC of CNLSMs $\mathrm{Ta}_{3} \mathrm{Y}$ compounds is much larger than that of $\mathrm{Nb}_{3} \mathrm{Y}$. Basically, the element Ta is heavier than Nb. Thus, the SOC in $\mathrm{Ta}_{3} \mathrm{Y}$ compounds may be stronger than those in $\mathrm{Nb}_{3} \mathrm{Y}$, leading to larger $\mathrm{SHC}$ in CNLSMs $\mathrm{Ta}_{3} \mathrm{As}$ family. Moreover, the SHC of $\mathrm{Ta}_{3} \mathrm{Bi}$ compound almost arrives to $1700(\hbar / e)(\Omega \cdot \mathrm{cm})^{-1}$ and becomes the largest one in the Dirac CNLSMs $\mathrm{X}_{3} \mathrm{Y}$ compounds, thanks to the larger atomic mass of element $\mathrm{Bi}$ than other members in the same group. In brief, the stronger $\mathrm{SHE}$ and larger $\mathrm{SHC}$ in $\mathrm{X}_{3} \mathrm{Y}$ family 
materials stem from larger atomic mass and stronger atomic SOC in these compounds. In addition, we show the same character of band structures and the effect of SOC on band inversion in different $\mathrm{X}_{3} \mathrm{Y}$ materials in Supplementary Fig. 8.

In summary, based on the first-principles calculations, we have predicted the $X_{3} Y(X=T a, N b ; Y=A s, S b, B i)$ family compounds as a kind of Dirac crossing nodal-line semimetal (CNLSMs) with large spin Hall conductivity (SHC), which can serve as an ideal platform to research rich topological quantum states and explore the application in spintronics. In the absence of SOC, three nodal lines converge at the $\mathrm{R}$ point and two lines cross with forming a nodal net around the $\Gamma$ point. After SOC is included, Dirac points are protected along the R-M path by the $C_{4}$ rotational symmetry and open a very tiny gap along other directions. The drumhead surface states conform to the feature of Dirac CNLSMs. At the same time, by calculating the momentum-resolved and bandresolved spin Hall conductivity, the large $\mathrm{SHC}$ in $\mathrm{Ta}_{3} \mathrm{As}$ is attributed to the SOC along X-M path, instead of the nodal points. This mechanism differs from both the Weyl point leading to SHC for $\mathrm{TaAs}$ and nodal line inducing $\mathrm{SHC}$ for $\mathrm{IrO}_{2}{ }^{9,14}$. The prediction in this work should promote further research on spin Hall effect, the topological and superconductivity properties, as well as quantum phase transitions. On the other hand, the fascinating crossing nodal-lines and the remarkable SHC at Fermi energy will contribute to their practical applications in assembling spintronic devices such as magnetic spin-orbit torque random access memory.

\section{METHODS \\ DFT calculations}

The first-principles calculations are performed within the framework of density functional theory (DFT), as implemented in QUANTUM ESPRESSO package ${ }^{42}$. The generalized gradient approximation in the parametrization of Perdew-Burke-Ernzerhof is used for the exchange-correlation potential $^{43}$. The kinetic energy cut-off and the charge density cut-off of the plane wave basis are chosen with 70 Ry and 850 Ry, respectively. All atoms are relaxed until the forces are smaller than $0.001 \mathrm{eV} / \AA \AA$. An $41 \times 41 \times 41 k$-point mesh in the electronic self-consistent calculations is used for the Brillouin zone (BZ) integration. To evaluate the thermodynamic stabilities of $X_{3} Y$ compounds, the formation enthalpies $(\Delta H)$ were calculated as: $\Delta H=\mathrm{E}$ $\left(X_{3} Y\right)-3 E(X)-E(Y)$. The phonon spectra were calculated using the PHONOPY codes based on the finite displacement method within a $4 \times 4 \times 4$ supercell with $3 \times 3 \times 3 k$-point mesh ${ }^{44}$. The spin Berry curvature, spin Hall conductivity, and topological edge states are calculated by using a tightbinding Hamiltonian constructed on a basis of maximally localized Wannier functions implemented in Wannier90 and WannierTools codes $^{45,46}$. The SHC is obtained by summing the spin Berry curvatures over all the occupied bands in a dense $k$-point grid of $200 \times 200 \times 200$.

\section{DATA AVAILABILITY}

The data that support the findings of this study are available from the corresponding author upon reasonable request.

Received: 25 December 2019; Accepted: 8 February 2021; Published online: 05 March 2021

\section{REFERENCES}

1. Safeer, C. K. et al. Room-temperature spin Hall effect in graphene/MoS 2 van der Waals heterostructures. Nano Lett. 19, 1074 (2019).

2. Feng, W., Yao, Y., Zhu, W., Zhou, J., Yao, W. \& Xiao, D. Intrinsic spin Hall effect in monolayers of group-VI dichalcogenides: a first-principles study. Phys. Rev. B 86, 165108 (2012)

3. Garcia, J. H., Cummings, A. W. \& Roche, S. Spin Hall effect and weak antilocalization in graphene/transition metal dichalcogenide heterostructures. Nano Lett. 17, 5078 (2017).
4. Sinova, J. et al. Universal intrinsic spin Hall effect. Phys. Rev. Lett. 92, 126603 (2004).

5. Jamali, M. et al. Giant spin pumping and inverse spin Hall effect in the presence of surface and bulk spin-orbit coupling of topological insulator $\mathrm{Bi}_{2} \mathrm{Se}_{3}$. Nano Lett. 15, 7126 (2015).

6. Lee, J. et al. Role of spin Hall effect in the topological side surface conduction. ACS Photonics 5, 3347 (2018).

7. Mellnik, A. R. et al. Spin-transfer torque generated by a topological insulator. Nature 511, 449 (2014).

8. Fan, Y. et al. Magnetization switching through giant spin-orbit torque in a magnetically doped topological insulator heterostructure. Nat. Mater. 13, 699 (2014).

9. Sun, Y., Zhang, Y., Felser, C. \& Yan, B. Strong intrinsic spin Hall effect in the TaAs family of Weyl semimetals. Phys. Rev. Lett. 117, 146403 (2016).

10. Guo, G. Y., Murakami, S., Chen, T. W. \& Nagaosa, N. Intrinsic spin Hall effect in platinum: first-principles calculations. Phys. Rev. Lett. 100, 096401 (2008).

11. Tanaka, T. et al. Intrinsic spin Hall effect and orbital Hall effect in $4 d$ and $5 d$ transition metals. Phys. Rev. B 77, 165117 (2008).

12. Zhou, J., Qiao, J., Bournel, A. \& Zhao, W. Intrinsic spin Hall conductivity of the semimetals $\mathrm{MoTe}_{2}$ and $\mathrm{WTe}_{2}$. Phys. Rev. B 99, 060408(R) (2019).

13. Pham, V. T. et al. Ferromagnetic/nonmagnetic nanostructures for the electrical measurement of the spin Hall effect. Nano Lett. 16, 6755 (2016).

14. Sun, Y., Zhang, Y., Liu, C.-X., Felser, C. \& Yan, B. Dirac nodal lines and induced spin Hall effect in metallic rutile oxides. Phys. Rev. B 95, 235104 (2017).

15. Chang, T. R. et al. Type-Il symmetry-protected topological Dirac semimetals. Phys. Rev. Lett. 119, 026404 (2017).

16. Yu, R., Weng, H., Fang, Z., Dai, X. \& Hu, X. Topological node-line semimetal and dirac semimetal state in antiperovskite $\mathrm{Cu}_{3}$ PdN. Phys. Rev. Lett. 115, 036807 (2015).

17. Gibson, Q. D. et al. Three-dimensional Dirac semimetals: design principles and predictions of new materials. Phys. Rev. B 91, 205128 (2015).

18. Wang, Z., Weng, H., Wu, Q., Dai, X. \& Fang, Z. Three-dimensional Dirac semimetal and quantum transport in $\mathrm{Cd}_{3} \mathrm{As}_{2}$. Phys. Rev. B 88, 125427 (2013).

19. Liu, J., Hou, W., Wang, E., Zhang, S., Sun, J.-T. \& Meng, S. Ideal type-Il Weyl phonons in wurtzite Cul. Phys. Rev. B 100, 081204(R) (2019).

20. Yu, R., Wu, Q., Fang, Z. \& Weng, H. From nodal chain semimetal to Weyl semimetal in $\mathrm{H}_{\mathrm{f}} \mathrm{C}$. Phys. Rev. Lett. 119, 036401 (2017).

21. Weng, $\mathrm{H}$. et al. Topological node-line semimetal in three-dimensional graphene networks. Phys. Rev. B 92, 045108 (2015).

22. Yamakage, A., Yamakawa, Y., Tanaka, Y. \& Okamoto, Y. Line-node Dirac semimetal and topological insulating phase in noncentrosymmetric pnictides $\mathrm{CaAgX}(\mathrm{X}=\mathrm{P}$, As). J. Phys. Soc. Jpn. 85, 013708 (2016).

23. Liu, B. et al. Flat AgTe honeycomb monolayer on Ag(111). J. Phys. Chem. Lett. 10, 1866-1871 (2019).

24. Du, Y. et al. Emergence of topological nodal lines and type-II Weyl nodes in the strong spin-orbit coupling system $\operatorname{lnNbX}_{2}(X=S$, Se). Phys. Rev. B 96, 235152 (2017).

25. Wu, W. et al. Nodal surface semimetals: theory and material realization. Phys. Rev. B 97, 115125 (2018).

26. Rui, W. B., Zhao, Y. X. \& Schnyder, A. P. Topological transport in Dirac nodal-line semimetals. Phys. Rev. B 97, 161113(R) (2018).

27. Xie, Q. et al. Phononic Weyl nodal straight lines in high-temperature superconductor $\mathrm{MgB}_{2}$. Phys. Rev. B 101, 024301 (2020).

28. Wang, J. et al. Pseudo Dirac nodal sphere semimetal. Phys. Rev. B 98, 201112(R) (2018).

29. Zhou, J., Qiao, J., Duan, C., Bournel, A., Wang, K. \& Zhao, W. Large tunneling magnetoresistance in $\mathrm{VSe}_{2} / \mathrm{MoS}_{2}$ magnetic tunnel junction. ACS Appl. Mater. Interfaces 11, 17647 (2019).

30. Aradhya, S. V., Rowlands, G. E., Oh, J., Ralph, D. C. \& Buhrman, R. A. Nanosecondtimescale low energy switching of in-plane magnetic tunnel junctions through dynamic oersted-field-assisted spin Hall effect. Nano Lett. 16, 5987 (2016).

31. Bracher, T. et al. Detection of short-waved spin waves in individual microscopic spin-wave waveguides using the inverse spin Hall effect. Nano Lett. 17, 7234 (2017).

32. Jin, M. J. et al. Nonlocal spin diffusion driven by giant spin Hall effect at oxide heterointerfaces. Nano Lett. 17, 36 (2017).

33. Lu, Z. W., Wei, S. \& Zunger, A. Electronic structure of ordered and disordered $\mathrm{Cu}_{3} \mathrm{Au}$ and $\mathrm{Cu}_{3}$ Pd. Phys. Rev. B 45, 10314 (1992).

34. Gubser, D. U., Hein, R. A., Waterstrat, R. M. \& Junod, A. Electronic and superconducting properties of the $\mathrm{Ti}_{3} \mathrm{P}$-type compounds $\mathrm{Nb}_{3} \mathrm{As}$ and $\mathrm{Nb}_{3}$ Si. Phys. Rev. $B$ 14, 3856 (1976)

35. Derunova, E., Sun, Y., Felser, C., Parkin, S. S. P., Yan, B. \& Ali, M. N. Giant intrinsic spin Hall effect in $W_{3} T a$ and other $A 15$ superconductors. Sci. Adv. 5, eaav8575 (2019). 
36. Fu, B. et al. Dirac nodal surfaces and nodal lines in ZrSiS. Sci. Adv. 5, eaau6459 (2019).

37. Wang, Z. et al. Dirac semimetal and topological phase transitions in $A_{3} B i(A=N a$, K, Rb). Phys. Rev. B 85, 195320 (2012).

38. Qiao, J., Zhou, J., Yuan, Z. \& Zhao, W. Calculation of intrinsic spin Hall conductivity by Wannier interpolation. Phys. Rev. B 98, 214402 (2018).

39. Guo, G. Y., Yao, Y. \& Niu, Q. Ab initio calculation of the intrinsic spin Hall effect in semiconductors. Phys. Rev. Lett. 94, 226601 (2005).

40. Liu, J., Meng, S. \& Sun, J. Spin-orientation-dependent topological states in twodimensional antiferromagnetic $\mathrm{NiTl}_{2} \mathrm{~S}_{4}$ monolayers. Nano Lett. 19, 3321 (2019).

41. Sui, X. et al. Giant enhancement of the intrinsic spin Hall conductivity in $\beta$-tungsten via substitutional doping. Phys. Rev. B 96, 241105(R) (2017).

42. Giannozzi, P. et al. QUANTUM ESPRESSO: a modular and open-source software project for quantum simulations of materials. J. Phys. Condens. Matter 21, 395502 (2009).

43. Perdew, J., Burke, K. \& Ernzerhof, M. Generalized gradient approximation made simple. Phys. Rev. Lett. 77, 3865 (1996).

44. Togo, A. \& Tanaka, I. First principles phonon calculations in materials science. Scr. Mater. 108, 1 (2015).

45. Wu, Q., Zhang, S., Song, H.-F., Troyer, M. \& Soluyanov, A. A. WannierTools: an open-source software package for novel topological materials. Comput. Phys. Commun. 224, 405 (2018).

46. Mostofi, A. A., Yates, J. R., Lee, Y.-S., Souza, I., Vanderbilt, D. \& Marzari, N. wannier90: a tool for obtaining maximally-localised Wannier functions. Comput. Phys. Commun. 178, 685 (2008).

\section{ACKNOWLEDGEMENTS}

The authors thank Bin Huang and Bin Cui for the helpful discussions. We acknowledge the National Key R\&D Program of China (2018YFB0407602), Beijing Municipal Science and Technology Project under Grant Z201100004220002, the Fundamental Research Funds for the Central Universities and the China Postdoctoral Science Foundation (No. 2020M670083, 2020M672039), the National Natural Science Foundation of China (Grant Nos. 61627813, 61571023, 11674139, 11674317, 11974348, 11834014, 11834005), the Strategic Priority Research Program of CAS (Grant No. XDB28000000 and No. XDB33000000), the National Key Technology Program of China (Grant No. 2017ZX01032101), the Special Foundation for Theoretical Physics Research Program of China (No. 11947207) for their financial support of this work. Parts of the calculations were performed at Tianhe2-JK at CSRC.

\section{AUTHOR CONTRIBUTIONS}

Z.Z., H.L., and W.Z. conceived and designed the project. W.H. performed all the DFT calculations, W.H. and J.L. did the theoretical analysis. All authors contributed to the manuscript writing.

\section{COMPETING INTERESTS}

The authors declare no competing interests.

\section{ADDITIONAL INFORMATION}

Supplementary information The online version contains supplementary material available at https://doi.org/10.1038/s41524-021-00504-w.

Correspondence and requests for materials should be addressed to Z.-G.Z., H.-G.L. or W.Z.

Reprints and permission information is available at http://www.nature.com/ reprints

Publisher's note Springer Nature remains neutral with regard to jurisdictional claims in published maps and institutional affiliations.

Access This article is licensed under a Creative Common Attribution 4.0 International License, which permits use, sharing, adaptation, distribution and reproduction in any medium or format, as long as you give appropriate credit to the original author(s) and the source, provide a link to the Creative Commons license, and indicate if changes were made. The images or other third party material in this article are included in the article's Creative Commons license, unless indicated otherwise in a credit line to the material. If material is not included in the article's Creative Commons license and your intended use is not permitted by statutory regulation or exceeds the permitted use, you will need to obtain permission directly from the copyright holder. To view a copy of this license, visit http://creativecommons. org/licenses/by/4.0/.

(c) The Author(s) 2021 